\title{
DESENVOLVIMENTO DE UM SOFTWARE PARA ENSINO E SIMULAÇÃO DE TRANSFERÊNCIA DE CALOR EM TEMPO REAL
}

\author{
DEVELOPMENT OF A SOFTWARE FOR TEACHING AND SIMULATION OF HEAT \\ TRANSFER IN REAL TIME \\ Josivan Pedro da Silva ${ }^{1}$, Danilo Emidio de Souza Cavalcanti ${ }^{2}$, Driele Karolaine dos Santos Costa $^{3}$, lago Jesus \\ de Souza ${ }^{4}$, Marcus Vinicius Santos de Moura ${ }^{5}$ \\ DOI: 10.37702/REE2236-0158.v40p300-309.2021
}

\section{RESUMO}

Este artigo tem como objetivo apresentar o desenvolvimento de um software para fins didáticos, no qual é possível estudar os processos de condução de calor através da visualização tridimensional. Uma vez que a maioria dos softwares semelhantes utilizam métodos como o de elementos finitos, que resultam em sistemas lineares complexos e de lenta resolução, o projeto utiliza uma abordagem analítica e dessa forma reduz drasticamente o tempo de simulações. Com o tempo reduzido é possível observar, em tempo real, esses processos os quais os docentes têm dificuldade em descrever, indo além das equações. Para as simulações são consideradas condições de contorno de temperatura e convecção. A partir do poder gráfico de um computador, é possível observar os diferentes comportamentos que dependem das características intrínsecas dos materiais, da sua geometria e das fontes de calor, assim proporcionando aos estudantes uma melhor compreensão de tais processos. Por consequência, facilita-se o entendimento dos fenômenos da transferência de calor e aumenta-se o engajamento e o interesse dos alunos.

Palavras-chave: Ensino de Engenharia; software de simulação; transferência de calor.

\begin{abstract}
This article aims to present the development of software for didactic purposes, where it is possible to study the processes of heat conduction through three-dimensional visualization. Since most software similar use methods finite element, which result in complex and slow-resolution linear systems, the project uses an analytical approach and, in this way, drastically reduces turnaround time. With the reduced time it is possible to observe, in real-time, these processes in which teachers find it difficult to describe, going beyond the equations. For the simulations, conditions are considered temperature contour and convection. Using the graphic power of a computer, it is possible to observe the different behaviors that depend on the intrinsic characteristics of materials, their geometry, and heat sources. Providing students with a better understanding of such processes. Consequently, facilitating the understanding of the transference phenomena heat and increasing student engagement and interest.
\end{abstract}

Keywords: Engineering Teaching; simulation software; heat transfer.

\footnotetext{
${ }^{1}$ Professor Doutor, Núcleo de Engenharia, UNINASSAU, Paulista-PE; josivan_silva@hotmail.com

${ }^{2}$ Coordenador e Professor Doutor, Núcleo de Engenharia, UNINASSAU, Paulista-PE; daniloescavalcanti@gmail.com

${ }^{3}$ Aluna de graduação, Núcleo de Engenharia, UNINASSAU, Paulista-PE.

${ }^{4}$ Aluno de graduação, Núcleo de Engenharia, UNINASSAU, Paulista-PE.

${ }^{5}$ Aluno de graduação, Núcleo de Engenharia, UNINASSAU, Paulista-PE.
} 


\section{INTRODUÇÃO}

Com o grande e constante avanço tecnológico, o mundo inteiro vem se adaptando a essa evolução. O ensino também foi se adaptando para a melhoria do acesso de seus usuários a conhecimentos sobre diversas áreas. As instituições de ensino também tiveram que se adaptar e ter suporte para trazer uma melhor conexão dos seus alunos a esse tipo de conteúdo. Com a chegada da pandemia e com as instituições fechadas, o uso de softwares e da internet se tornaram indispensáveis para dar andamento aos cronogramas escolares.

A dificuldade de aprendizagem pode estar relacionada a diversos fatores culturais, sociais e biológicos, mas não indica falta de motivação ou inteligência para aprender. Nos cursos de Engenharia uma dessas barreiras é a capacidade de abstração e visualização tridimensional dos efeitos físico-químicos (FLOREZ; LEBRÃO; LEBRÃO, 2017). Muitas vezes essa contextualização visual fica ainda mais prejudicada por estar associada a conceitos matemáticos complexos. Tal dificuldade pode ocorrer devido ao déficit ferramental matemático no histórico de desenvolvimento educacional do aluno (BARBETA; YAMAMOTO, 2002).

Com o avanço das tecnologias de visualização $3 \mathrm{D}$ o processo chamado de gameficação tem ganhado muitos adeptos. Esse processo se baseia em captar a atenção dos alunos utilizando ferramentas atrativas e interativas, semelhantes a jogos (MORSCHHEUSER et al., 2018; SILVA; SOUZA; SILVA, 2013; SILVA et al., 2016). Entre os diversos pontos positivos desse processo se tem que processos simulados por software permitem reduzir o custo com laboratórios e aulas experimentais, cobrindo possíveis deficiências nesse aspecto (BOGUSEVSCHI; MUNTEAN; MUNTEAN, 2020). Tudo isso facilita o processo de aprendizagem dos alunos e, como dito por Vicente et al., (2021), com a ajuda da internet e do avanço dos computadores, ficou mais acessível o desenvolvimento de softwares que permitam essa interação.
Tendo isso em vista, há que se considerar ainda que diversos conceitos são complexos e de difícil entendimento dentro da engenharia, como os relacionados aos processos de transferência de calor. Este trabalho se propõe a demonstrar o desenvolvimento de um software que permite a visualização de processos de transferência de calor para facilitar o aprendizado deste conteúdo na área da engenharia. O software visa a uma demonstração em tempo real desses processos em 3D, utilizando o poder computacional de uma unidade de processamento gráfico (GPU), comumente chamada de placa de vídeo, permitindo a aplicação de soluções analíticas, no caso as equações de Green, as quais dependem das características do material, da geometria e das temperaturas às quais o objeto de estudo está sujeito (SILVA, OLIVEIRA, FILHO, 2020).

O software resultante deste trabalho foi desenvolvido utilizando-se o Unity $3 \mathrm{D} \AA$ como ambiente de desenvolvimento, pois este é gratuito até se atingir um certo lucro e tem disponibilidade para desenvolvimento de aplicativos para outras plataformas, se desejado, o que torna o Unity $3 \mathrm{D} \AA$ acessível a todo o público interessado. Tudo isso permite a criação de uma base para o desenvolvimento da programação referente às simulações: "a utilização destas ferramentas em sala de aula leva indubitavelmente a um maior entendimento da física envolvida no processo em questão, sem que aluno tenha de gastar tempo com exaustivas manipulações algébricas" (MUNIZ; MARCZAK, 2002).

\section{FUNDAMENTAÇÃO TEÓRICA}

\section{Software}

Com o avanço da sociedade, principalmente no aspecto científico e tecnológico, os softwares se tornaram onipresentes em vários aspectos do nosso dia a dia; eles podem se apresentar de várias formas, como um aplicativo no celular ou até um programa de computador, jogos em consoles entre outros. Os softwares são basicamente uma sequência de instruções, que é interpretada e 
executada por um dispositivo de processamento, gerando assim um resultado útil ao usuário. Na engenharia não é diferente, a otimização e organização dos processos industriais leva à constante introdução dos aplicativos e programas, muitos dos quais utilizados na didática educacional (JUCÁ, 2006).

\section{Softwares Educacionais}

Programas de computadores educacionais são cada vez mais usados por educadores e alunos no auxílio da aprendizagem. A introdução da tecnologia na sala de aula de maneira intuitiva torna mais fácil a aderência a algumas disciplinas. A visualização de objetos em 3D, o uso de plataformas de reuniões remotas, jogos educativos entre outros estão cada vez mais presentes em todo processo educacional, desde o ensino básico até o superior. (MACHADO, 2016; FIALHO; MATOS, 2010).

\section{Transferência de Calor}

O termo utilizado popularmente como "calor" pode ser definido como um tipo de energia de movimentação das moléculas, que pode ser transportado de um corpo para o outro quando estes não estão em equilíbrio térmico. Ou seja, sempre que existir uma diferença na temperatura de qualquer um dos elementos haverá transferência de calor (SCHMIDT; HENDERSON; WOLGEMUTH, 1996). Sendo assim, essa mudança térmica pode ocorrer de três formas - condução, convecção e radiação , podendo ser descrita de forma numérica ou analítica. Este trabalho visa a gerar resultados de forma analítica utilizando as equações de Green, que representam a solução fundamental da equação de calor quando se utiliza como condição inicial uma fonte de calor de temperatura e posição conhecida. Definida da seguinte forma:

$$
\left\{\begin{array}{c}
u_{t}(\mathrm{x}, \mathrm{t})-\mathrm{k} u_{x x}(\mathrm{x}, \mathrm{t})=0 \\
u(\mathrm{x}, 0)=\delta(\mathrm{x})
\end{array}\right.
$$

Sendo $(\mathrm{x}, \mathrm{t}) \in \square>0 \mathrm{e}^{\delta(\mathrm{x})}$ a função delta de Dirac, apresenta solução do tipo:

$$
u(\mathrm{x}, \mathrm{t})=\frac{1}{\sqrt{4 \pi k t}} e^{\left(-\frac{x^{2}}{4 k t}\right)}
$$

Essa equação 2 pode ser generalizada para dimensões maiores, assim como para situações mais gerais. $\mathrm{O}$ estudo da transferência de calor pode ser encontrado em simulações de equipamentos industriais, tais como: processos de aquecimento e resfriamento de peças mecânicas, processos sujeitos a forte atrito, dissipadores de calor e processos químicos (GARCIA, 2005).

\section{Materiais e métodos}

Além do uso do computador convencional para a criação de peças $3 \mathrm{D}$ e $\mathrm{o}$ acompanhamento do processo de condução térmica em tempo real, fez-se necessário o uso de programação, com o uso de instruções computacionais para a criação de um aplicativo com fins didáticos, a partir do qual, de maneira intuitiva, o usuário possa acompanhar a transferência térmica visualmente através de um gradiente de cores dispostos em peças 3D.

Entre os softwares utilizados para o desenvolvimento deste trabalho destacam-se os seguintes:

a) 3Ds Max ${ }^{\circledR}$;

b) Unity $3 \mathrm{D} \AA$.

O 3Ds Max® é um programa de modelagem 3D que permite renderização de imagens e animações, muito usado em produção de filmes de animação e criação de personagens de jogos e é também utilizado para a criação das peças de análise (ROMEIRO,2018). São necessárias análises de escala, texturização dos objetos, permitindo ao usuário a customização da peça, entre outros cuidados que deverão ser tomados durante o fluxo de trabalho. Com isso, os objetos virtuais em 3D estarão prontos para aplicação no software a ser desenvolvido. Esse software tem sido utilizado no desenvolvimento de diversos trabalhos na área de engenharia e educação 
(SILVA; SOUZA; SILVA, 2011; SILVA; SOUZA; SILVA, 2013; SILVA et al., 2016).

As peças, depois de ganhar forma no processo do 3Ds Max®, segue para o Unity $3 \mathrm{D} \AA$, que é uma plataforma de desenvolvimento de jogos e aplicativos educacionais em tempo real, criada em 2005, a qual oferece ao usuário a capacidade de criação em 2D e 3D, utilizando a programação em C\#. Diversos trabalhos na literatura têm utilizado esse software na área de educação e engenharia (SILVA, SOUZA， SILVA，2011; SILVA, SOUZA, SILVA, 2013; SILVA et al., 2016), este ambiente de desenvolvimento tem como suas principais características:

a) facilidade no acesso;

b) interface amigável;

c) acesso gratuito para estudantes;

d) desenvolvimento para diversas plataformas, eentre elas: IOS, Linux, Windows, Android, Xbox, Playstation.

Além da modelagem em 3D dos objetos, para a criação do aplicativo foi utilizado o Shaderforge, que é uma ferramenta que permite a criação de shaders dentro do Unity $3 \mathrm{D} \AA$, que definem como os materiais serão visualizados. Dessa forma, ele é o responsável pela visualização do gradiente de cores através do uso das equações de Green.

\section{RESULTADOS E DISCUSSÃO}

O desenvolvimento de softwares para a área educacional é algo que tem crescido ao longo do tempo, principalmente devido às aulas remotas impostas pela pandemia (SILVA; FILHO, 2020). Porém, os softwares no mercado têm aplicação restrita quando se trata de determinados assuntos. Para aprendizado dos fenômenos de transferência de calor, por exemplo, os softwares disponíveis são simuladores como Ansys ${ }^{\odot}$, SimScale $^{\odot}$, $\mathrm{Comsol}^{\odot}$ entre outros. Esses softwares apresentam uma curva de aprendizado muito lenta; para um aluno de graduação entender o funcionamento destes seria necessária uma disciplina inteira para isso. Ademais, esses softwares requerem um tempo de simulação muitas vezes proibitivo para aplicação em sala de aula, além de terem custo elevado. Levando em consideração tais pontos, o presente trabalho tem como propósito apresentar o desenvolvimento de um software de simulação de processos de transferência de calor em tempo real, utilizando soluções analíticas para se atingir esse objetivo. Todo o processo começa com a modelagem 3D do objeto a ser simulado.

\section{Modelagem 3D}

Os objetos a serem simulados precisam ser modelados em um software 3D, o software escolhido foi o 3Ds Max $^{\odot}$. Outros softwares poderiam ser utilizados, como o Autodesk inventor ou Solidworks, porém o 3Ds Max tem larga aplicação no desenvolvimento de softwares interativos. A partir da composição de formas geométricas simples é possível se obter peças complexas (Figura 1).

Figura 1 - Interface do 3Ds $\operatorname{Max}^{\oplus}$, demonstrando uma peça modelada

Fonte: acervo dos autores.

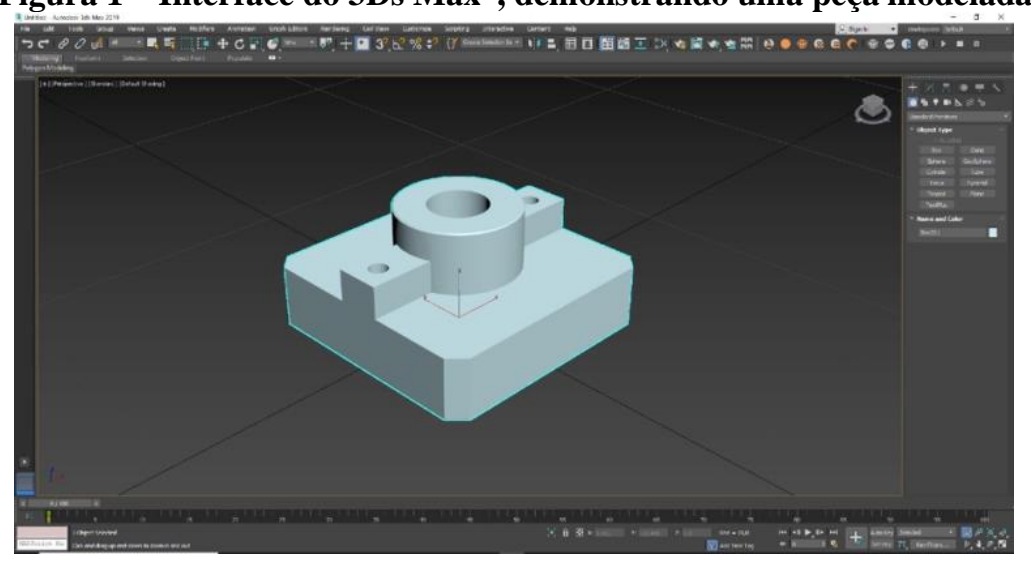


Na Figura 1 é possível observar a interface do 3Ds $\mathrm{Max}^{\odot}$, que, através de operações de adição e subtração de formas simples, como cilindros e paralelepípedos, permite a geração de peças de engenharia. Uma das vantagens do 3Ds $\operatorname{Max}^{\odot}$ é possibilitar a utilização da modelagem poligonal, ao contrário dos softwares de CAD, nos quais se utiliza modelagem paramétrica. Dessa forma, é possível manter a quantidade de polígonos baixa na peça, tornando o programa desenvolvido viável em dispositivos menos poderosos. A peça, na Figura 1, é uma presilha de vedação e atende a especificação necessária para o método analítico de transferência de calor, que é ser uma peça majoritariamente convexa. Após a confecção da peça, o modelo é exportado para o ambiente de desenvolvimento: o Unity $3 \mathrm{D}^{\odot}$.

\section{Engine de desenvolvimento}

Para desenvolvimento do software foi utilizado o Unity $3 \mathrm{D}^{\odot}$, devido a sua acessibilidade e facilidade. Após um novo projeto ter sido criado no Unity $3 \mathrm{D}^{\odot}$, foi possível transferir para sua pasta de trabalho todos os arquivos necessários como modelos e texturas, como o que foi desenvolvido na Figura 1. Após transferidos, eles são reconhecidos imediatamente e podem ser incluídos em uma cena. Além destes, diversos componentes ainda precisam ser adicionados, conforme exposto na Figura 2:

Figura 2 - Interface do Unity 3D@, demonstrando uma cena do software desenvolvido

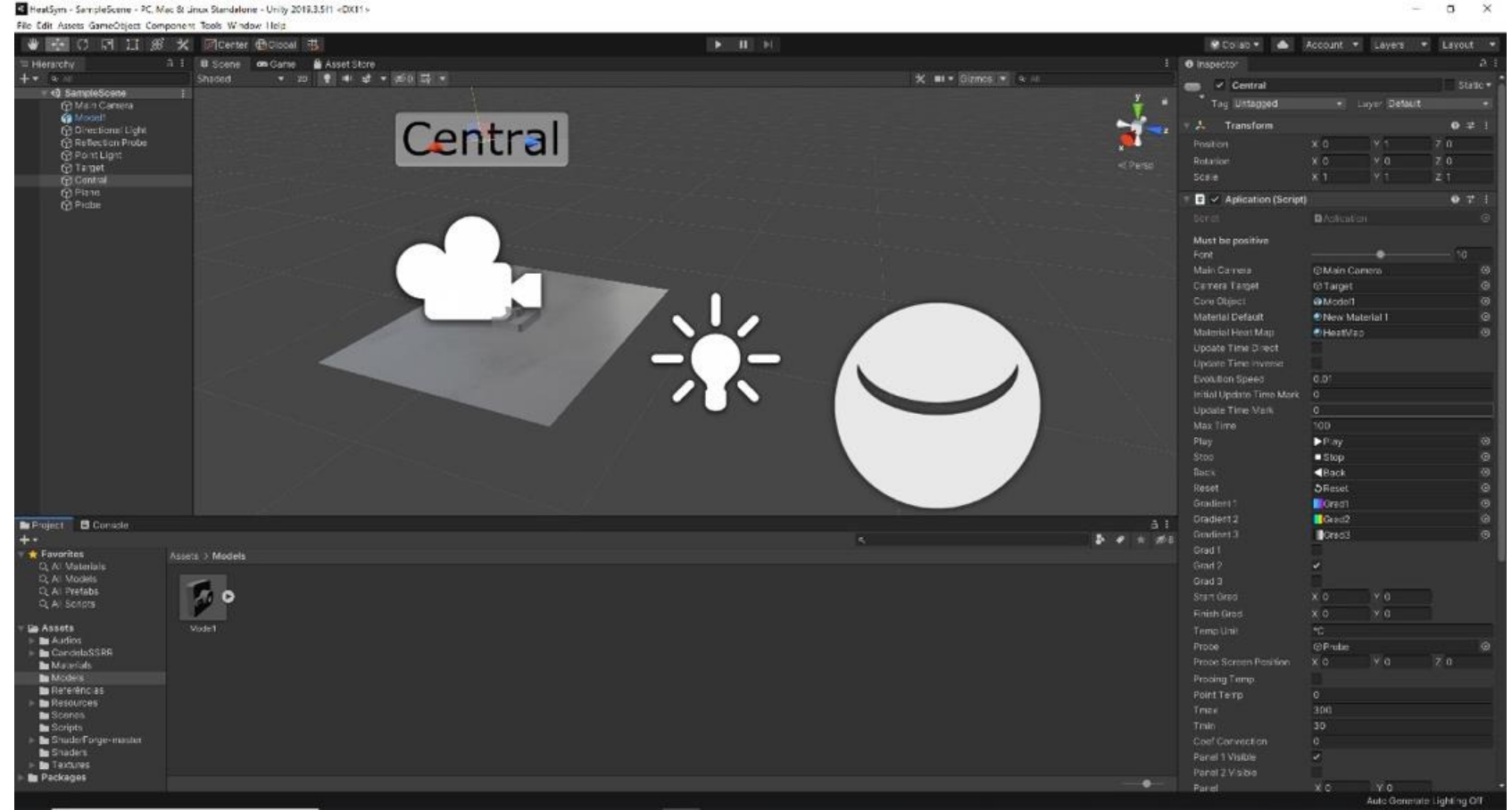

Fonte: acervo dos autores.

A Figura 2 demonstra o Unity $3 \mathrm{D}^{\odot}$ e os componentes utilizados no desenvolvimento do software deste trabalho. Além dos modelos serem incluídos é necessário incluir uma câmera, ela que permite a geração do ponto de vista, o qual será mostrado ao usuário do software. Atrelada a câmera ainda é necessária a adição de scripts que permitem a movimentação desta, a partir da interação do usuário via mouse e teclado, além de gerar a interface do software desenvolvido. A iluminação também precisa ser adicionada, assim como também as sondas de reflexão (esfera branca na Figura 2). Tais sondas permitem a geração de reflexos que permitem um maior realismo, principalmente em peças metálicas. Na interface visível do Unity $3 \mathrm{D}^{\odot}$, na Figura 2, é possível observar a lista de objetos adicionados à cena, na parte superior esquerda, e a lista de pastas do projeto, na parte inferior 
esquerda. Os conteúdos dessas pastas podem ser visualizados na parte central inferior da Figura 2. Os detalhes do objeto selecionado podem ser vistos na parte lateral direita. Informações como posição e scripts adicionados ficam visíveis nesse local.

O ponto simulação entra na criação dos materiais que irão ser aplicados sobre a peça; ao menos dois tipos de materiais ficam disponíveis para a peça, o material que descreve o comportamento visual da peça e um material que descreve como a transferência de calor está se desenvolvendo. O material que representa o visual existe por padrão no Unity $3 \mathrm{D}^{\odot}$, a única necessidade é ajustar os parâmetros para atingir uma visualização realista. Porém, o material que descreve a transferência de calor teve que ser construído do zero, pois as equações de
Green precisam ser aplicadas e visualizadas. Um material nada mais é do que um conjunto de parâmetros aplicados a um shader, que guarda a programação que vai definir como um objeto é visualizado. Shaders envolvem conceitos matemáticos e de programação que são muito complexos e normalmente são desenvolvidos em ambientes de construção via nós, agrupando blocos. Dessa forma, é possível desenvolver algo mais visual e intuitivo. Diversas são as ferramentas para isso, porém uma delas se destaca pela solidez e documentação. O Shaderforge é um plug-in para criação de shaders no Unity $3 \mathrm{D}^{\odot}$. As equações de Green foram aplicadas sobre a programação do shader, gerando um fluxograma como o que se segue (Figura 3):

Figura 3 - Nós para a criação do shader para visualização do processo de condução de calor

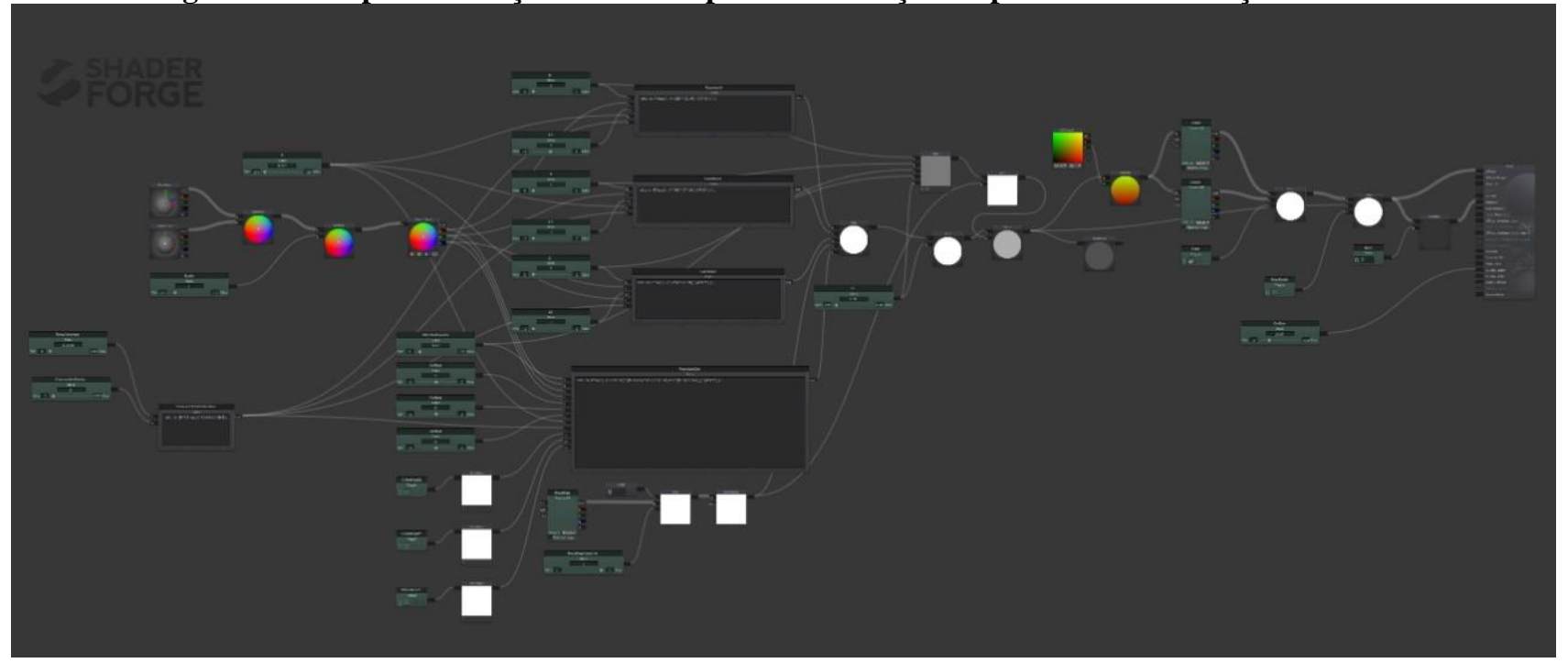

Fonte: acervo dos autores.

Na Figura 3 é possível observar o fluxo de nós criados para a visualização do gradiente de calor gerado nas simulações. Da esquerda para a direita os nós vão seguindo a composição das regras matemáticas que definem esse shader. Inicialmente, mais à esquerda, tem-se diversas entradas, como o tempo e a constante de condutividade, assim como as posições relativas de cada ponto da peça. Próximo ao centro, tem-se parâmetros que influenciam como a fonte de calor se comporta, de forma plana, linear ou pontual. Ainda próximo ao centro, tem-se retângulos cinzas que representam as equações de Green, definidas na forma de código. Essas equações geram saídas numa escala de cinza, que é então remostada para um gradiente de escolha do usuário. Dessa forma, a visualização da escala de temperatura pode ser visualizada, como exposto na Figura 4. 


\section{Software desenvolvido}

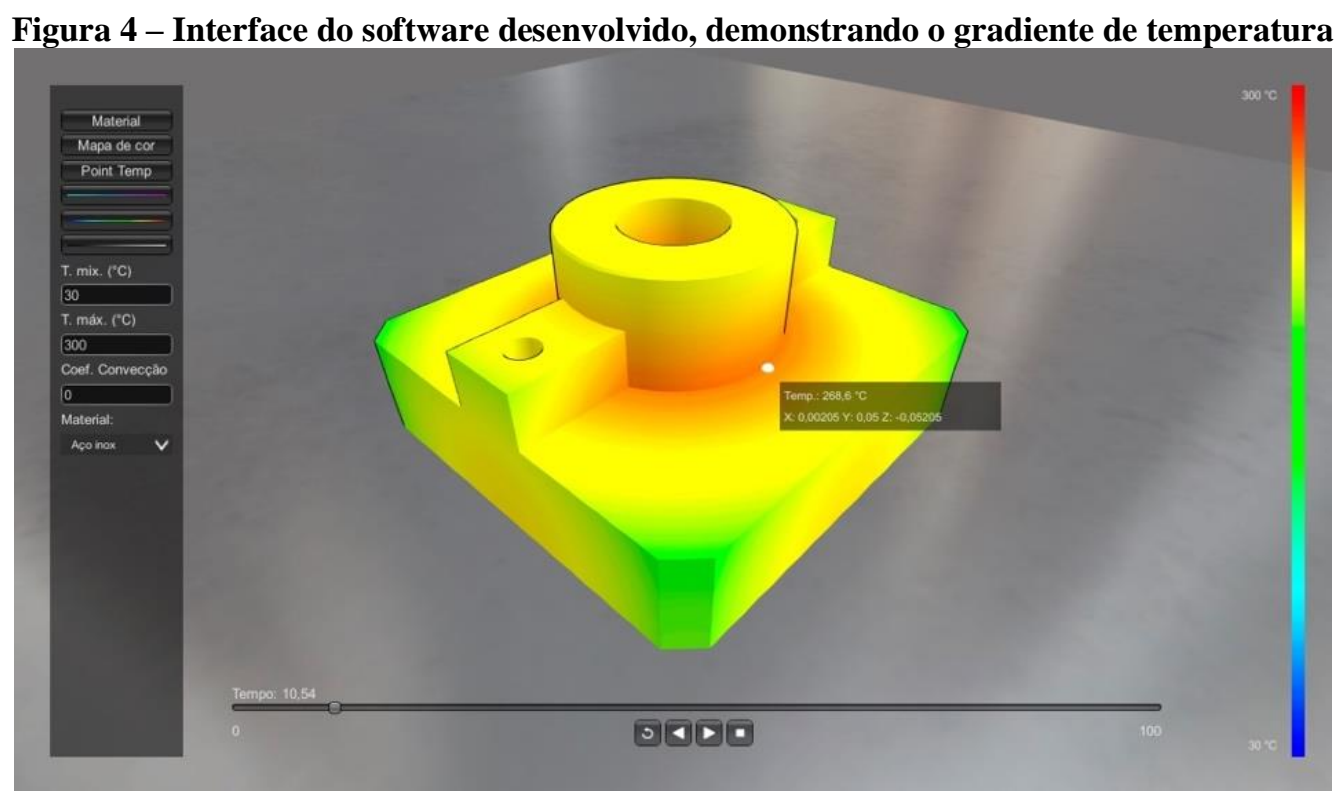

Fonte: acervo dos autores.

Graças ao Shader demonstrado na Figura 3 foi possível desenvolver a visualização da Figura 4. No centro da Figura 4 é possível observar a peça de estudo com um gradiente que demonstra a escala de cor representada na parte direita da Figura, indo do valor mínimo, em azul $\left(30^{\circ} \mathrm{C}\right)$, até o valor mais alto, em vermelho $\left(300^{\circ} \mathrm{C}\right)$. Além disso, é possível observar a interface final desenvolvida para o software; na parte esquerda é possível visualizar as informações de entrada, por exemplo, permitindo o usuário visualizar o material real da peça ou o gradiente de cores. É possível também sondar a temperatura em um ponto específico, como demonstrado no centro da Figura 4. Ademais, o menu permite diversas possibilidades como definir que gradiente utilizar, temperaturas mínimas e máximas, coeficiente de convecção e tipo de material. Além deste um outro menu foi criado, para definir as condições das regiões de geração de calor, e outro, na parte inferior, definido para demonstrar as características de avanço no tempo, permitindo a simulação em tempo real. O usuário tem liberdade para explorar a simulação em qualquer instante de tempo, assim como reiniciar, parar, retroceder, entre outras possibilidades. A evolução no tempo pode ser estudada variando-se diversos parâmetros, dessa forma é possível obter algo como o que se vê na Figura 5.
Figura 5 - Evolução no tempo, demonstrando a condução de calor

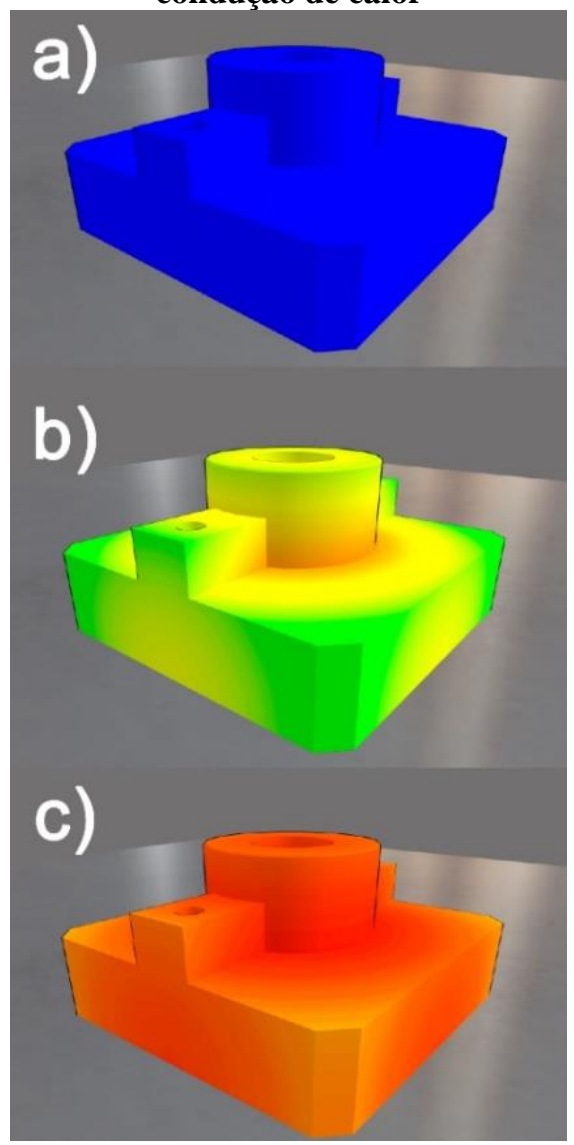

Fonte: acervo dos autores.

A Figura 5 demonstra a condução de calor existente na peça quando uma fonte pontual é aplicada na parte central inferior, a escala respeita a faixa de temperatura definida na 
Figura 4. Para intervalos de tempo diferentes é possível observar como a condução se dá - indo de a) até c) -, tudo sendo visualizado em tempo real, sem necessidade de esperar a simulação ocorrer.

\section{CONSIDERAÇÕES FINAIS}

Conforme citado anteriormente, este artigo trata de um projeto voltado para apresentação de um software de simulação em tempo real, o qual pode ser utilizado para o ensino de transferência de calor na engenharia. A interação visual facilita a compreensão e permite que os beneficiados assimilem mais rapidamente os processos, viabilizando um entendimento empírico do assunto. Por se tratar de um simulador de um processo comum em diversas áreas, o benefício é interdisciplinar, podendo ser útil a diversos cursos, como na análise da transferência de calor em uma peça de uma linha de produção ou uma simulação termográfica de peças mecânicas em atrito. Contudo, ainda é um assunto que carece de pesquisas. Em projetos futuros, pode-se desenvolver outros pontos, tornando a experiência cada vez mais completa. É possível acrescentar mais modelos geométricos, possibilitando uma maior diversidade no comportamento da condução de calor. Além disso, o software foi desenvolvido para análises da transferência de calor através da condução e convecção. Expandir o programa e adicionar outro tipo de condução de calor, como a radiação, tornaria o software adequado a mais situações.

\section{AGRADECIMENTOS}

Os autores agradecem o apoio do Núcleo de Engenharia da UNINASSAU, localizado em Paulista-PE, pela concessão de bolsas de pesquisa e disponibilização da estrutura de apoio.

\section{REFERÊNCIAS}

BARBETA, V. B; YAMAMOTO, I. Dificuldades conceituais em física apresentados por alunos ingressantes em um curso de engenharia. Rev. Bras. Ensino Fís., v. 24, n. 3, São Paulo, 2002.

BOGUSEVSCHI, D.; MUNTEAN, C.; MUNTEAN G.M. Teaching and Learning Physics using 3D virtual learning environment: A case study of combined virtual reality and virtual laboratory in secondary school. Journal of Computers in Mathematics and Science Teaching, Waynesville, NC EUA, v. 39, n. 1, p. 5-18, 2020.

FIALHO, N. N; MATOS, E. L. N. A arte de envolver o aluno na aprendizagem de ciências utilizando softwares educacionais. Educar em Revista, Curitiba, n. especial 2, p. 121-136, 2010.

FLOREZ, B. M; LEBRÃO G. W; LEBRÃO S. M. G. A aprendizagem ativa com uso de impressão tridimensional no curso de engenharia. International Journal on Active Learning, Rio de Janeiro, v. 2, n. 1, p. 79-85, jan./jun., 2017.

GARCIA, C. Modelagem e Simulação de processos industriais e de sistemas eletromecânicos, São Paulo: EDUSP, 2005.

JUCÁ, S. C. A relevância dos softwares educativos na educação profissional. Ciências \& Cognição, CEFET-CE, Fortaleza, Ceará, v. 8, p. 22-28, 2006.

MACHADO, A. S. Uso de softwares educacionais, objetos de aprendizagens e simulações no ensino de química. Revista Química Nova na Escola, v. 38, n. 2, p. 104-111, 2016.

MARCZAK, L. D. F.; MUNIZ, A. R. Uma aplicação do software maple no ensino de transferência de calor. Anais... IX CONGRESSO BRASILEIRO DE ENGENHARIA E CIÊNCIAS TÉRMICAS, Caxambu-MG, 2002.

MORSCHHEUSER, B. et al. How to design gamification? A method for engineering gamified software. Information and Software Technology, v. 95, p. 219-237, 2018.

ROMEIRO, N. C. Digitalização de peças anatômicas para utilização em mídias 3D. 2018. 45 f. TCC (Graduação) - Curso de Design, Departamento de Expressão Grafica, 
Universidade Federal de Santa Catarina, Florianópolis, 2018.

SCHMIDT, F.W; HENDERSON, R.E; WOLGEMUTH, C.H. Introdução às ciências térmicas, termodinâmica e transferência de calor. São Paulo: Edgard Blucher,1996.

SILVA, J. P.; SOUZA, T. P. C.; SILVA, J. M. F. Aplicação de programas interativos 3d Para o ensino da termodinâmica. Anais... VI Congresso brasileiro de termodinâmica Aplicada, CBTERMO, Salvador, Bahia, 2013.

SILVA, J. P.; SOUZA, T. P. C.; SILVA, J. M. F. Estudo da utilização de softwares alternativos para visualização e modelagem de poços petrolíferos. Anais... $6^{\circ}$ Congresso brasileiro de pesquisa e desenvolvimento em petróleo e gás, PDPETRO, Florianópolis, Santa Catarina, 2011.

SILVA, J. P. et al. Desenvolvimento de software 3D para controle de processos, aplicado a produção de biodiesel. Anais... Congresso Brasileiro de Engenharia Química, COBEQ, Ceará, Fortaleza, 2016.

SILVA, M.; OLIVEIRA, G.; FILHO, R. Plataforma computacional para análise da Transferência de calor bidimensional. Revista de Ensino de Engenharia, v. 39, p. 390-399, 2020.

SILVA, J. P. B; FILHO, D. M. L. Software educacionais e suas aplicações em tempos de pandemia: estudo sobre possibilidades de aplicação. Brazilian Journal of Development, v. 6, n. 7, p. 50866-50878, 2020.

VICENTE, A. R. et al. Desafios da educação infanto-juvenil: os efeitos da Covid-19. Rev. Eletrônica Pesquiseduca. Santos, v.13, n. 29, p. 386-398, jan.-abr., 2021.

\section{DADOS BIOGRÁFICOS DOS AUTORES}
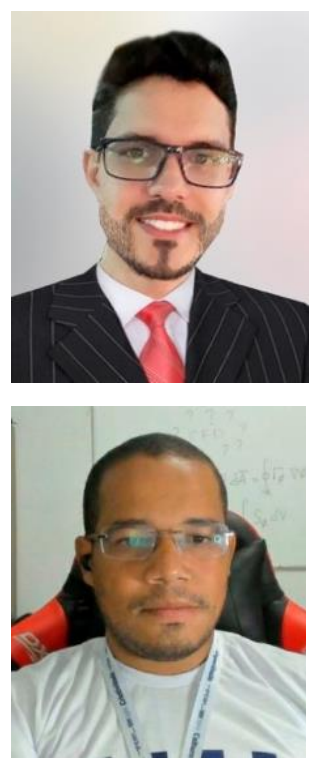

Josivan Pedro da Silva - Possui doutorado, mestrado e graduação no Departamento de Engenharia Química (DEQ), da Universidade Federal de Pernambuco (UFPE), em 2018, 2013 e 2010, respectivamente. Desenvolveu trabalhos no Laboratório de Simulação e Dinâmica de Processos Químicos (LSDPQ), tendo experiência nas áreas de modelagem matemática e 3D, simulação, programação, automação, inteligência artificial, processos industriais, processos oxidativos avançados e biocombustíveis. Foi Professor no Centro Universitário Estácio do Recife. Atualmente é Professor na UNINASSAU, Unidade Paulista-PE.

Danilo Emidio de Souza Cavalcanti - Possui doutorado, mestrado e graduação no Departamento de Engenharia Química (DEQ), da Universidade Federal de Pernambuco (UFPE), em 2018, 2013 e 2011, respectivamente. Na Engenharia Química tem experiência nas áreas de Geoquímica, no estudo da dinâmica do fósforo em reservatórios, Processos Oxidativos Avançados, Minerais Industriais, no desenvolvimento de estudos na calcinação de gipsita, utilizando Fluidodinâmica Computacional (CFD) e modelagem de Processos de Reforma Seca e DesidroAromatização. Foi Professor Titular II no Centro Universitário Estácio do Recife. Foi Professor Substituto do Departamento de Engenharia Mecânica da Universidade Federal de Pernambuco (DEMEC-UFPE). Foi coordenador dos cursos de Engenharia de Produção, Elétrica, Mecânica e Civil no Centro Universitário Joaquim Nabuco - Uninabuco Paulista. Atualmente é coordenador dos cursos de Engenharia de Produção, Elétrica, Mecânica e Civil no Centro Universitário Maurício de Nassau - UniNassau Paulista e Professor Permanente do Mestrado Profissional em Tecnologia Ambiental do Instituto de Tecnologia de Pernambuco (ITEP-PE). 


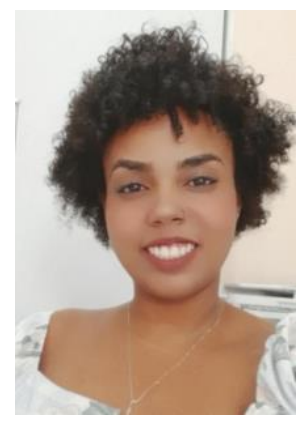

Driele Karolaine dos Santos Costa - Graduanda em Engenharia de Produção, formada no curso Técnico em Segurança do Trabalho (2016), tem experiência na área de Segurança do trabalho e supervisora de produção, com ênfase na área de gestão de pessoas e controle de qualidade.

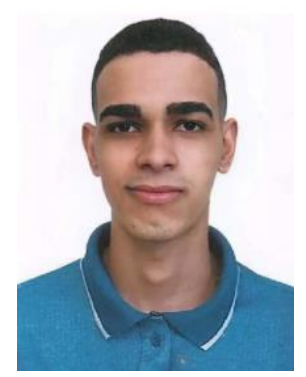

Iago Jesus de Souza - Graduando em Engenharia Mecânica na UNINASSAU, possui experiência na área de computação, matemática e monitoria de cálculo. Atualmente trabalha como auxiliar de escritório na Água Mineral Rosa Branca Montania LTDA. 